\title{
Douleur, corps, travail : Fatima, une clinique de la complexité
}

\author{
Pain, Body, Work: Fatima, a Clinical Approach of Complex Situations
}

\author{
M. Pezé \\ (C) Lavoisier SAS 2020
}

\begin{abstract}
Résumé L'analyse de la réciprocité des influences entre les registres corporels, psychiques et sociaux de l'être humain est l'objet même d'une clinique de la complexité, sans que l'on puisse dégager une instance ultime qui serait la clé explicative de l'ensemble : l'inconscient, le cerveau, les gènes, le caractère, les relations familiales, la généalogie, le milieu social, le travail et son organisation. Chacun de ces déterminants est à la fois conditionné et conditionnant, produit et producteur. La clinique de la complexité consiste à puiser dans les outils de pensée des uns et des autres pour construire des solutions cliniques.
\end{abstract}

Mots clés Clinique de la complexité · Division sexuelle du travail $\cdot$ Deuxième corps $\cdot$ Centralité du travail

\begin{abstract}
The analysis of the reciprocity of influences between the physical, mental and social registers of the human being is the very object of a clinic of complexity, without it being possible to identify an ultimate authority that would be the key to explaining the whole: unconscious, brain, genes, character, family relationships, genealogy, social environment, work and its organization. Each of these determinants is at the same time conditioned and conditioning, produced and productive. The clinic of complexity is about drawing on each other's tools of thought to construct clinical solutions.
\end{abstract}

Keywords Complexity clinic - Sexual division of labor · Second body $\cdot$ Centrality of work

L'analyse de la réciprocité des influences entre les registres corporels, psychiques et sociaux de l'être humain est l'objet même d'une clinique de la complexité, sans que l'on puisse dégager une instance ultime qui serait la clé explicative de l'ensemble : l'inconscient, le cerveau, les gènes, le caractère, les relations familiales, la généalogie, le milieu social, le travail et son organisation. Chacun de ces déterminants est

\section{Pezé $(\square)$}

Association diffusion des connaissances sur le travail humain, 22, rue Édouard-Vaillant, F-92300 Levallois-Perret, France e-mail :mmpeze@gmail.com à la fois conditionné et conditionnant, produit et producteur. La clinique de la complexité consiste à puiser dans les outils de pensée des uns et des autres pour construire des solutions cliniques.

Dans la prise en charge de la douleur, quid de la place faite au travail ? Le travail est une rencontre entre qui nous sommes et ce qu'on nous donne à faire :

Il serait illusoire de penser que nous laissons notre histoire personnelle accrochée sur un cintre, dans les vestiaires de notre lieu de travail. Le travail est un des pôles majeurs d'expression de l'identité, et on ne peut le réduire à la simple exécution de la tâche prescrite. Faire l'impasse sur le sens du travail reviendrait à en dénier le caractère d'activité humaine mobilisant non seulement les compétences intellectuelles et/ou manuelles du sujet mais aussi sa personnalité tout entière. La plupart des sujets en bonne santé espèrent avoir l'occasion, grâce au travail, de s'accomplir, d'accéder à une reconnaissance de leur valeur.

Si le salarié s'investit trop au travail, on pourra donc émettre l'hypothèse qu'il a un besoin éperdu de reconnaissance non obtenue dans l'enfance. Le lien difficile de certains salariés à l'autorité peut toujours être travaillé sous l'angle de la relation à la figure paternelle. Mais peut-on dire à l'ouvrière qui souffre des 27 bouchons qu'elle visse par minute que son œdipe y est pour quelque chose ? Peut-on dire au harcelé qui s'effondre à son poste, « mais, pourquoi n'êtes-vous pas parti plus tôt au lieu de supporter cette souffrance ?», alors que démissionner lui aurait fait perdre ses droits sociaux ? Les femmes apportent-elles leur consentement pulsionnel à être payées $25 \%$ de moins que les hommes?

Quand le choix d'un métier est conforme aux besoins du sujet et que ses modalités d'exercice permettent le libre jeu $\mathrm{du}$ fonctionnement mental et corporel, nous savons que le travail peut occuper une fonction centrale dans l'équilibre psychosomatique. Mais si l'individu, de par la pauvreté manuelle ou mentale de sa tâche, de par l'hyperprocéduralisation de l'organisation de son travail, ne peut pas bien travailler, il y aura souffrance.

Le travail use les corps : muscles, tendons, synoviales, microvascularisation, nerfs. Ces pathologies sont regroupées 
sous le sigle de «TMS », troubles musculosquelettiques, qui est une création hybride. Hormis les médecins du travail, les ergonomes et préventeurs et quelques équipes médicales spécialisées, le sigle TMS est largement absent des diagnostics médicaux.

Chaque spécialité médicale a son intitulé diagnostique et thérapeutique pour ces pathologies :

- en rhumatologie, on s'intéresse à la fibromyalgie ;

- en chirurgie de la main, on opère des affections localisées (hygroma du coude, canal carpien, etc.) ;

- en médecine de la douleur, on parle de tableau douloureux chronique, pas de TMS.

Cette diversité des disciplines complexifie les diagnostics et le repérage des facteurs à l'œuvre dans l'apparition de ces lésions. Le travail en tous cas porte atteinte aux supports anatomiques du mouvement.

Le travail artistique, artisanal, est précédé par une élaboration mentale et devient donc activité d'expression. Là, le geste est riche et mobilise le corps au service du sens. Dans d'autres types de travail, le geste se résume à une simple décharge motrice. L'organisation du travail détermine le contenu et les procédures de la tâche, fixe même les modalités des relations entre les sujets en assignant à chacun place et rôle par rapport aux autres travailleurs. Quelquefois, le fonctionnement mental est réservé aux uns, le fonctionnement corporel assigné aux autres.

Dans le réseau de consultations « Souffrance et Travail » créé depuis 1995, nous traitons toutes les pathologies, organiques et psychiques.

Dans les corps abîmés de nos patients est toujours déposée un peu de l'identité transgénérationnelle d'abord. Comme cet ouvrier qui ne se remettait pas d'être vivant après qu'un mur lui soit tombé dessus et qui transportait avec lui, en plus du sachet plastique rempli des médicaments, une enveloppe marron contenant des certificats, des médailles. Celles de son père, de son grand-père, harkis, qui s'étaient battus pour la France et avaient été honorés pour leur courage. Je lis à voix haute les certificats de bravoure. Là, il n'est plus sous les gravats du mur mais dans son histoire.

Dans les corps abîmés de nos patients est toujours déposée un peu de leur identité de genre. Une femme ne bouge pas comme un homme, ne travaille pas comme un homme, n'a pas les mêmes emplois qu'un homme, d'ailleurs.

Dans les corps abîmés de nos patients est toujours déposée un peu de la souffrance du deuxième corps, le corps imaginaire, subjectif, réduit au « silence mental ». L'absence de signification, l'inutilité des gestes à accomplir façonnent une image de soi terne, enlaidie, misérable, une fatigueusure du geste vidé de sens mais qu'il faut accomplir quand même.

Objectivité oblige, la souffrance mentale, la fatigue sont irrecevables au travail. Seule la maladie physique peut être entendue et bénéficie d'un statut de réalité. La prise en charge médicale va achever de déplacer la souffrance mentale vers la douleur physique.

\section{L'histoire de Fatima}

Le service de pathologies professionnelles de l'hôpital Raymond-Poincaré de Garches nous adresse Fatima E., 48 ans. La première phrase de la lettre d'accompagnement scelle son destin : « la patiente est d'origine marocaine, illettrée, souffre de sinistrose ». Effectivement, femme de ménage pour cinq employeurs différents (école maternelle, cages d'escalier dans deux groupes d'immeubles différents, ménage chez deux particuliers), Fatima est soumise à de nombreux déplacements entre des sites professionnels espacés.

En janvier, elle a fait une chute dans un escalier et se plaint depuis de douleurs multiples de tout l'hémicorps gauche que les différents rhumatologues consultés ne s'expliquent pas. Les nombreux examens complémentaires pratiqués (radiographies, scanner, scintigraphie osseuse, EMG, IRM) sont en décalage avec la plainte douloureuse. Au bout d'un an, sans diagnostic étayé, le médecin-conseil a consolidé l'accident du travail et suspendu le versement des indemnités journalières. C'est le début de la précarité.

On nous l'envoie avec l'alternative diagnostique habituelle : sinistrose ou fibromyalgie? Fatima est enfoncée dans un tableau douloureux chronique avec son cortège de prises en charge multiples et disparates, de traitements commencés, vite arrêtés, de difficultés administratives avec la sécurité sociale, ses employeurs et les créanciers. Le discours est enfoncé dans une plainte corporelle majeure ; la souffrance morale est de tonalité dépressive, l'épuisement est patent.

Il faut déplier le tableau clinique.

Fatima signale des douleurs préexistantes à l'accident mais dont elle ne tenait pas compte, engagées dans du « tenir », soucieuse de maintenir son autonomie financière. Car à l'épuisement physique évident de la patiente s'ajoute l'usure morale d'élever seule deux filles depuis son divorce. Venue en France avec son mari, sans formation, ne lisant et n'écrivant pas le français, elle n'a pu trouver que du travail ménager.

L'étude des différents examens complémentaires permet d'additionner des pathologies modérées isolément, mais dont le cumul explique l'ampleur de la plainte douloureuse : séquelles d'ostéite de la jambe gauche dans l'enfance, uncodiscarthrose $\mathrm{C} 4-\mathrm{C} 5$, C5-C6, scoliose lombaire, bascule pelvienne, pincement discal L4-L5, neuropathie sensitive L5-S1, protrusion L4-L5, L5-S1, pathologie dégénérative en $\mathrm{L} 2$, séquelle de phlébite à la jambe gauche. Les douleurs sont permanentes, majorées par toute position prolongée, assise, debout, allongée. Elles réagissent un peu aux antalgiques, au chaud, au repos. 
Fatima revient longuement sur la chute dans l'escalier qu'elle a vécu comme "un événement qui a tout fracassé de l'intérieur ", goutte d'eau qui a fait déborder le vase et remis en cause un équilibre préexistant précaire. Les cauchemars répétitifs, la peur de tomber à nouveau ont été présents pendant deux mois, signant une névrose traumatique passée inaperçue. La multiplication des prises en charge, toutes organicistes, a entraîné l'invisibilité du tableau de PTSD. La patiente, très intuitive et capable d'insight, ajoute : « $a u$ bout de deux mois, la douleur a remplacé la peur». Le tableau douloureux chronique qui s'est mis en place est une transformation de l'effraction psychique en maladie organique.

L'approche d'un tel tableau devenant incontournablement pluridisciplinaire.

Fatima est confiée au Dr Adeline, médecin de la douleur, anesthésiste-réanimateur, pour la mise en place d'un traitement adéquat. Son classement travailleur handicapé est immédiatement demandé auprès de la MDPH locale. La non-communication d'éléments médicaux cohérents au médecin-conseil avait entraîné une situation de doute clinique sur le cas de Fatima et provoqué la suspension du versement des indemnités. Le médecin-conseil n'a pas tous les éléments médicaux, il faut les lui transmettre et reprendre un dialogue. Une reprise d'un travail est compromise, il va donc falloir construire aussi un statut social à cette femme pour éviter la bascule définitive de toute une famille dans la précarité. Nous savons que prescrire des antalgiques et démarrer une psychothérapie avec un patient dans la précarité est illusoire. La stabilisation de la situation sociale est plus efficace que la prescription d'antidépresseur et l'analyse de la névrose infantile.

Nous continuons donc inlassablement les démarches administratives. Les contacts répétés avec le médecinconseil débouchent sur son accord pour une invalidité de type I compatible avec un futur travail à mi-temps. L'invalidité obtenue permet la prise en charge du prêt bancaire par l'assurance souscrite par Fatima. L'inscription aux Assedic pour une recherche d'emploi ne peut être obtenue faute d'un licenciement de la part des cinq employeurs différents. Après contact avec un des médecins du travail, une inaptitude définitive est prononcée. Mais c'est à moi qu'il revient de rédiger les nombreux courriers afin d'obtenir de chaque employeur un certificat de licenciement légal, car Fatima ne sait pas rédiger en français. Au terme de ces longues démarches fastidieuses, Fatima voit sa précarité stabilisée.

Je vais enfin pouvoir commencer mon travail.

Fatima peut enfin me parler de son enfance. Elle est née au Maroc, troisième fille d'une fratrie nombreuse, de parents commerçants. En dépit de résultats prometteurs à l'école, Fatima ne s'est arrêtée que parce que l'institutrice française demandait un sou par faute d'orthographe, pris sur le budget nourriture de sa mère et qu'elle ne voulait pas lui rendre la vie plus difficile. Seuls les fils ont fait des études dans sa famille. Ils sont procureur, magistrat, ingénieur.

On l'a marié avec un homme beaucoup plus âgé, bûcheron, qui l'emmène en France et ne trouve aucun travail. Elle décide de travailler pour assurer la survie de ses deux filles qui sont nées rapidement. Elle devient femme de ménage.

La prise en charge de la saleté, les tâches simples, répétitives, monotones nécessitent minutie, patience et rapidité mais aussi un sens éthique de la nécessaire prise en charge du réel :

"Il faut bien le faire », répète Fatima, « Je tiens la maison de la femme qui travaille. Grâce à moi, elle travaille la tête libre ".

Si ses différentes identités ont d'évidence été confisquées et même trahies par sa famille, son mari, ses employeurs, l'espace de la psychothérapie ramènent le travail psychique sur le devant de la scène. Fatima raconte l'invisibilité de ses savoir-faire de femme de ménage. Son Verbe est écouté, reconnu, encouragé. La verbalisation des tensions va vite transformer la douloureuse économie musculaire en dynamique psychique.

Fatima s'étonne de penser. Elle me demande si elle a le droit. Un jour, bouleversée, elle arrive à sa séance et m'annonce :

- La nuit, je me relève et j'écris. Les mots me viennent sans que je ne puisse rien y faire. C'est une douleur et une jouissance. Je voudrais vous dire ce que j'ai écrit.

- Alors, venez avec vos écrits à la prochaine séance!

Commence alors une étrange chorégraphie entre la patiente, qui arrive avec ses grandes feuilles de cahier noircies, et moi, la thérapeute qui ne lit pas l'arabe. Phrase après phrase, nous cherchons ensemble la traduction la plus juste. Elle démarre la traduction, bute sur le mot français qu'elle ne trouve pas, je lui demande quelle idée elle veut exprimer, elle me donne une image, je propose des mots et le pas de deux produit des feuillets qui s'accumulent.

Feuille après feuille, j'écris sous la dictée « le livre de Fatima ». Le livre de la petite fille dont l'accès au savoir a été interrompu, dont le corps et la psyché ont été encastrés dans des comportements féminins prédestinés. Le livre de la femme découvrant un mari faible, dont il faut pallier les carences tout en respectant sa domination. Le livre de l'immigrée qui nettoie la maison des femmes qui travaillent, dans un double effacement, celui de ses compétences, celui de ses origines.

Si les séquelles musculosquelettiques sont irréversibles, l'expression du deuxième corps permet une deuxième naissance. "Le livre de Fatima » devient une œuvre à part entière n'appelant aucune interprétation ou commentaire théorique. 
14 février :

"Je suis fatiguée de mes pensées, de ma faiblesse devant la force que demande la vie; je suis fatiguée du tribunal qui marche dans ma tête et mon cour. Je suis fatiguée d'avoir peur pour mes filles, fatiguée de ma solitude; En 16 ans, je $n$ 'ai pas connu le repos un jour, une heure. En 16 ans, je n'ai pas connu ma féminité un jour, une heure.

Il faut que je me dépêche de prendre une décision avant que je sois trop faible. Je ne veux plus vivre dans la peur et l'humiliation. Je suis comme un livre. Toutes les femmes sont des livres dont le titre est le mari. Prenez le temps d'ouvrir les livres. ")

27 janvier :

La langue de Fatima se résume à : "oui, merci » en souriant. Elle prend grand soin de ce qu'on lui confie et fait du bon travail. Elle sait que si elle n'a pas de diplômes, elle a le sourire, le oui et le merci. On peut lui faire confiance.

Les jours passent, premier enfant. Fatima ne sait pas quoi faire. Avec un bébé, il y a des besoins.

Fatima est debout à 4 heures 30 le matin. Elle réveille l'enfant, la lave, l'habille, la porte sur son dos. Elle prend son sac à main et son manteau sous le bras, sort de chez elle à 4 heures 45.

Un deuxième enfant. Les besoins grandissent. Fatima continue à s'occuper de son travail, de sa famille, seule. Son corps et son cour s'usent au fil des années. Abimée, Fatima ne travaille plus.

14 février :

Le travail d'écriture permet une amélioration nette du niveau de la douleur. Le travail psychique a porté sur les ressorts de la domination masculine dans sa culture, sur les compétences nécessaires au travail du ménage. Fatima parle au nom des artistes du quotidien :

Le talent est si probant, la poésie si puissante, que toute l'équipe se mobilise pour aider Fatima.

« Au nom de Dieu, ou de leur travail, ou du devoir, ils sont venus jusqu'à mon âme et l'ont sorti du coin. Elle n'était plus seule. Le Dr Adeline est très patient avec moi. Il s'occupe du cassé. Mme Pezé est très patiente avec moi. Elle cherche mon âme battue par les vents. Comment ont-ils transformé mon âme peureuse en une âme pleine d'espoir? Mon âme est dans un corps cassé, il ne reste qu'une main en état.

Ils ne me donnent pas l'espoir, le courage et point final. Ils me donnent envie de devenir comme eux. Je prends mon crayon et je vais écrire comme eux. Ce n'est pas un grand crayon mais j'écris comme eux.

Un jour, à l'aube, j'ai pris un crayon, une feuille, j'ai allumé la lampe et j'ai écrit. Pendant des semaines, je n'ai pas dormi la nuit pour écrire dans ma tête. J'ai écrit mes pensées pour ne pas qu'elles se perdent.
J'ai trouvé enfin ce qui c'était perdu en moi. Avec ces deux médecins qui m'ont cherché. Le torchon dans une main et le crayon dans l'autre. "

Je suis bouleversée de ce retour de tout le travail accompli, de tous les obstacles à soulever, des centaines de séances consacrées à cette femme et de l'avènement de cette identité puissante et créatrice. Elle ne sait pas que pour l'aider à s'affranchir d'autant de leviers de soumission, la psychanalyse ne m'a pas suffi comme socle de pensée. Elle ne sait pas les longues heures de discussion que j'ai eues sur son cas avec Danièle Kergoat, directrice de recherche au CNRS, à l'origine des concepts de division sexuelle du travail et de ce qu'elle appelle la position féminine fautive. Elle ne sait pas ce que notre travail psychothérapique doit à Pascale Molinier, maître de conférences à la chaire de psychologie du travail à l'époque, spécialiste du travail sur le genre.

Fatima ne sait pas que faute de papier, c'est dans un autre hôpital que sera photocopié son manuscrit en plusieurs exemplaires. Armée de ses manuscrits dans des sacs plastiques, Fatima part au Salon du Livre et, de stand en stand, les donne à lire. C'est l'éditeur Bacharri, en lisant quelques pages, qui va mesurer la puissance poétique de cette femme. La chaîne ne va plus s'interrompre autour d'elle. Prière à la lune de Fatima Elayoubi sort en 2006. Zoé Varier, journaliste, lui consacre une émission sur France Inter, ouvre même une souscription pour financer ses études. Véronique Maurus, grand reporter, lui consacre un magnifique portrait dans le Monde « héroïne du ménage ». Tant d'autres se joindront à cette chaîne pour que Fatima réalise son rêve : étudier. Elle est entrée à l'université Paris-X-Nanterre, en DAEU en septembre 2007. C'est avec la fébrilité de tous les écoliers qu'elle a acheté sa trousse, ses crayons, ses cahiers, choisi les couleurs pour les matières et qu'elle a affronté les grands amphis, les professeurs, les étudiants, tous beaucoup plus jeunes qu'elle. Mais Fatima ne craint plus rien ni personne. Fatima dit qu'elle est arrivée en France illettrée et qu'elle a enfin trouvé la Lettre. Elle va placer la lettre française à côté de la lettre arabe et pourra ainsi bientôt écrire directement en français. Elle montera sur scène au Festival de Cannes avec l'actrice qui joue son rôle dans le film « Fatima » de Philippe Faucon qui remportera le césar du meilleur film en 2016.

$\mathrm{Si}$, dans un premier temps, le travail perd nos patients, c'est bien le travail qui les sauve. Le travail thérapeutique d'abord, sur leur psyché et leur corps. Le travail avec l'équipe de soins où chacun a joué une part active dans le retour à l'emploi. Un emploi enfin ou une activité et un statut social, qui leur a rendu une place parmi les autres et l'usage de leur corps. Pour Fatima, le travail de l'écriture.

Plutôt que d'évacuer le territoire du travail comme étranger à l'approche thérapeutique, il apparaît plus fructueux d'entendre ce que les patients ont à dire en les « suivant» dans leurs ateliers, usines, bureaux, etc. On perçoit alors la centralité du travail dans les enjeux de la construction identitaire dès lors 
que les regards des autres, pairs et supérieurs, jouent leur rôle narcissiste ou laminant. La clinique de la complexité impose de ne rien s'interdire pour traiter nos patients.

Liens d'intérêts : l'auteur déclare ne pas avoir de lien d'intérêt.

\section{Bibliographie}

1. Dejours C (1993) Travail usure mentale. De la psychopathologie à la psychodynamique du travail. Bayard Éditions, Paris

2. Elayoubi F (2006) Prière à la lune. Ed Bacharri, Paris
3. Justins DM (1997) Syndrome douloureux régional complexe (SDRC). In: Brasseur L, Chauvin M, Guilbaud G (eds) Douleurs. Maloine, Paris

4. Hirata H, Kergoat D (1988) Rapport sociaux de sexe et psychopathologie du travail. In: Plaisir et souffrance au travail. Tome II. AOCIP, Paris, pp 131-63

5. Marty $P$ (1991) Genèse des maladies graves et critères de gravité en psychosomatique. Revue française de psychosomatique. PUF, Paris, pp 5-22

6. Mauss (1936) Les techniques du corps. Sociologie et Anthropologie. PUF, Paris, pp 365-88

7. Pezé M (2002) Le deuxième corps. La Dispute, Paris

8. Pezé M (2008) Ils ne mouraient pas tous mais tous étaient frappés. Pearson, Paris 